\title{
The veridical perception of object temperature with varying skin temperature
}

\author{
MARK F. TRITSCH \\ Johannes Gutenberg University, Mainz, West Germany
}

\begin{abstract}
The effect of skin-adaptation temperature on object-temperature perception was investigated, using the method of dichiric matching, in an attempt to determine whether veridical perception of physical object temperature occurs in human subjects. Observers were presented with a test temperature on one hand and required to find a matching temperature, that is, one that produced the same sensation, on the other, differently adapted, hand. Using equality of test and matching temperatures as a criterion of veridical perception, it was found that the latter improves with $\Delta T$, the difference between object temperature and skin-adaptation temperature. It is postulated that when $\Delta T$ is close to zero, veridical perception of object temperature is sacrificed to allow for improved performance in distinguishing object materials, whereas at high positive and negative values of $\Delta T$, veridical perception is preserved so that object temperature can be correctly identified. A mechanism is proposed in which veridical perception can be achieved by means of parallel processing of static and dynamic temperature information.
\end{abstract}

Several classical experiments in the psychophysics of temperature sensations have shown that varying the adaptation temperature of the skin can result in substantial error in the perception of object temperature. For example, in the "three-bowls experiment,"1 the left and right hands are immersed for several minutes in bowls containing water at adapting temperatures of $10^{\circ}$ and $40^{\circ} \mathrm{C}$, respectively. When both hands are then placed in a third bowl containing water at $27^{\circ} \mathrm{C}$, the left hand feels distinctly warm and the right hand feels distinctly cool (Hering, 1877). The same effect is obtained when both hands are placed on a water-circulated brass thermode at $27^{\circ} \mathrm{C}$. Thus, an object of a given temperature can produce either warm or cold sensations when it is touched.

The three-bowls experiment was interpreted by early experimenters as demonstrating that object-temperature sensations do not provide information about the absolute temperature of an object (Frey, 1904). Indeed, Weber (1846) had already concluded, from essentially similar experiments, that only change in temperature was an adequate stimulus. However, versions of the three-bowls experiment reported by Goldscheider (1926) and Hahn (1929), who used different combinations of adaptation and test temperature, produced results inconsistent with this view, and suggested that there might be some veridical perception of object temperature. Before the present experiments were conducted, pilot experiments, in which variants of the three-bowls experiment were investigated, confirmed these results. The test temperature of the third bowl was systematically varied within the range of $13^{\circ}$

This article represents a portion of the doctoral dissertation submitted by the author to Johannes Gutenberg University. The author's mailing address is Institute of Zoology (Biophysics Section), Johannes Gutenberg University, Postfach 3980, 6500 Mainz, West Germany. to $46^{\circ} \mathrm{C}$, while the two adapting temperatures were set at $20^{\circ}$ and $42^{\circ} \mathrm{C}$. It was found that the same sensation occurred in both hands when the test temperature was higher or lower than both of the adapting temperatures. In between, the amount of difference in sensation varied, appearing greatest between $23^{\circ}$ and $33^{\circ} \mathrm{C}$ when one hand felt warm and the other cold. Similar results were obtained when the experiment was carried out separately for each hand, using magnitude estimates as a measure of sensation, ${ }^{2}$ and when the test bath was replaced with a watercirculated thermode. Thus, under some experimental conditions that differ from the ones conventionally employed in the three-bowls experiment, object-temperature sensations do not seem to be influenced by the adaptation temperature of the skin.

The experiments reported below were designed to provide quantitative data on the relationship between skinadaptation and object temperatures and the sensations of warmth and cold. The method adopted can be described as the dichiric matching of temperature stimuli. Its essential features are the following: (1) Each hand of the observer is adapted at a different temperature. (2) The observer is presented with a test temperature to one hand, and then requested to find a temperature stimulus that produces the same sensation (i.e., a matching temperature) with the other hand. (3) Deviations from veridical perception of object temperature are revealed by differences between test temperature and matching temperature.

Two experiments were carried out. Experiment 1 was concerned with investigating an underlying assumption of dichiric matching, that is, that, under the conditions of the experiments, the adaptation temperature of one hand has no influence on temperature sensation in the contralateral hand. This was tested by using magnitude estimation of temperature sensations on one hand and vary- 
ing the adaptation temperature of the other. In Experiment 2, the dichiric method was employed with various combinations of adaptation and object temperature to answer the principal question posed in the study.

\section{EXPERIMENT 1}

\section{Method}

Observers. Fifteen male students were paid to take part as observers in this experiment. They had had no previous experience in the experimental method employed, and were not informed of the aim of the investigation.

Apparatus. Two interchangeable, thermostatically controlled water baths were used to adapt the left hand of the observer to a preset temperature $\left(T_{\mathrm{Al}}\right)$ of $31.5^{\circ}$ or $40.5^{\circ} \mathrm{C}$. A submerged mitten made of very thin plastic foil was attached to each water bath. The observer could insert his left hand into the mitten and remove it again without difficulty, ensuring that the hand remained dry throughout the experiment. The $31.5^{\circ} \mathrm{C}$ water bath was also connected to a small brass-topped thermode which set the adaptation temperature for the fingers of the right hand $\left(T_{\mathrm{Ar}}\right)$. The objecttemperature stimuli $\left(T_{\mathrm{O}_{\mathrm{r}}}\right)$ consisted of 10 small aluminum disks (40 $\mathrm{mm}$ in diameter and $8 \mathrm{~mm}$ thick), maintained at a given temperature by being clamped onto appropriate positions along the aluminum temperature column described in detail in Experiment 2. The values of $T_{\mathrm{Or}}$ investigated were $33.5^{\circ}, 34.8^{\circ}, 36.1^{\circ}, 37.4^{\circ}$, $38.9^{\circ}, 40.3^{\circ}, 41.5^{\circ}, 42.7^{\circ}, 43.7^{\circ}$, and $44.8^{\circ} \mathrm{C}$. The temperatures were continuously monitored by using a digital thermometer that could be switched to read from any of 10 thermistors clamped onto the disks. Each disk had a small wooden handle so that during its application as a stimulus it could be removed from its clamp and placed against the skin. Air temperature varied between $22^{\circ}$ and $24^{\circ} \mathrm{C}$ during the experiment.

Procedure. Each observer attended a single experimental session consisting of two stimulus series. During the first series, $T_{\mathrm{Al}}$ was $31.5^{\circ} \mathrm{C}$; during the second, it was $40.5^{\circ} \mathrm{C}$. The task of the observer was subjective magnitude estimation with free choice of modulus. The observer sat on a chair with the adaptation bath at his left side and the thermode on a table in front of him. On his right side was a screen with a small hole through which the right hand could be inserted. The screen prevented the observer from seeing the stimulus object used.

At the beginning of the session, the observer was presented with written instructions explaining the experimental procedure and how to perform the magnitude estimations. As soon as any questions had been dealt with, the observer's left hand was immersed in the $31.5^{\circ} \mathrm{C}$ adaptation bath, while the first, second, and third fingers of the right hand were placed, volar surface down, on the brasstopped thermode. After a 15-min preadaptation period, the first test of the first series was carried out. The observer lifted both hands from their respective adaptation positions, rested the left hand, palm upward, on a cushion and inserted the right hand through the opening in the screen. The experimenter placed the stimulus disk against a marked area on the volar surface of the first, second, and third fingers for $2 \mathrm{sec}$, after which the observer estimated the sensation. Both hands were then returned to their adapting positions. This was followed, in $45 \mathrm{sec}$, by the next test.

Following completion of the first series of tests, the left hand was transferred to the $40.5^{\circ} \mathrm{C}$ adaptation bath. After a preadaptation period of $25 \mathrm{~min}$, tests in the second series were carried out just as in the first series. Each series consisted of 30 tests divided into three consecutive groups of 10 . Each group of tests consisted of the 10 object temperatures presented in random order.

\section{Results and Discussion}

The results showed that warm adaptation of the hand has no effect on the sensation of warmth in the contralateral hand. In Figure 1 subjective magnitude estimates, $E$ (geometric average of all 15 observers), are plotted against the object-temperature stimulus, $T_{\mathrm{Or}}$, for both adaptation temperatures, $T_{\mathrm{Al}}$. No significant difference can be detected between the two sets of results. Most observers tended to shift their modulus following the second period of adaptation. The shift showed no preferred direction, indicating that it was caused by observer difficulty in remembering the modulus originally chosen. As a result, however, the variance of $E$ is higher for the second series than for the first (average standard deviation of the normalized log estimates was 0.16 in the second series but only 0.13 in the first). ${ }^{3}$

It can be concluded that there are no contralateral effects of adaptation that might lead to error in the method of dichiric matching used in Experiment 2. The absence of such effects contrasts with the well-documented bilateral summation of both threshold and suprathreshold stimuli in the sensations of warmth and cold (Hardy \& Oppel, 1937; Marks \& J. C. Stevens, 1973; Rózsa \& Kenshalo, 1977). Presumably, the adaptation that affects object-temperature perception occurs peripherally to the point in the CNS where bilateral summation occurs.

To check on whether or not these conclusions would be valid for the adaptation temperature range between $15^{\circ}$ and $30^{\circ} \mathrm{C}$, the following experiment was carried out. The adaptation procedure employed earlier could not be repeated, since exposure of the entire hand to $15^{\circ} \mathrm{C}$ induces an oscillating pattern of vasodilation and vasoconstriction (Lewis reaction), making it impossible to control adaptation temperature. Instead, the volar surface of

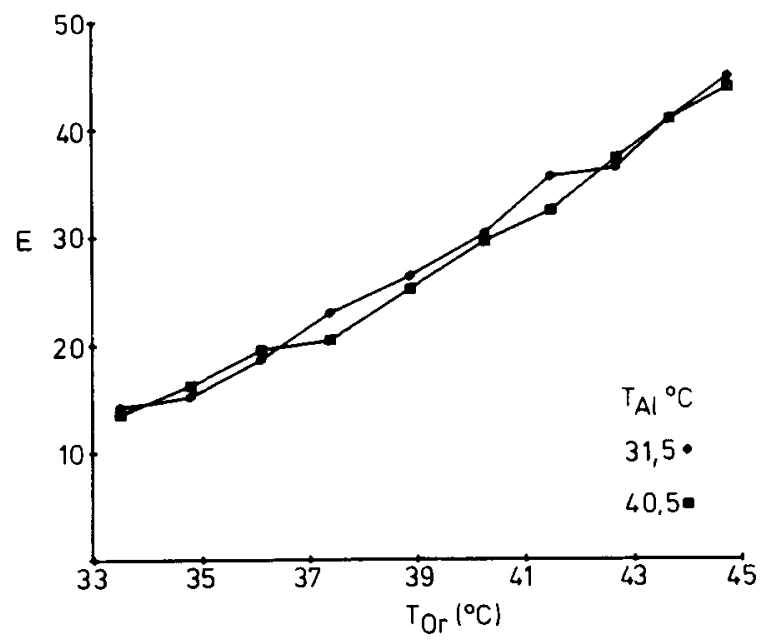

Figure 1. Subjective magnitude estimates, $E$, at two different adaptation temperatures, $T_{\mathrm{Al}}$, of the contralateral hand. Test temperature $T_{O r}$ is shown on the horizontal axis. 
the index and middle fingers of each hand were adapted directly on an aluminum temperature column.

Changes in the adaptation temperature of one hand which affect sensation in the other would also be expected to alter the second hand's indifference temperature, that is, the temperature at which neither cold nor warm sensations are felt. Three observers were asked to identify this temperature on the temperature column, using the fingers of the right hand adapted at $15^{\circ} \mathrm{C}$. With each observer, 20 such tests were made with each of the two adaptation temperatures used for the left hand. The results showed that there were no significant bilateral effects. With a $15^{\circ} \mathrm{C}$ adaptation temperature on the left hand, the mean indifference temperature chosen with the right hand was $18^{\circ} \mathrm{C}\left(S E M=0.16^{\circ} \mathrm{C}\right)$. When the left hand was adapted to $30^{\circ} \mathrm{C}$, the mean indifference temperature chosen was $17.7^{\circ} \mathrm{C}\left(S E M=0.27^{\circ} \mathrm{C}\right)$.

\section{EXPERIMENT 2}

\section{Method}

Observers. The 9 paid observers ( 4 male and 5 female) were students aged between 21 and 32 years and without previous experience as experimental subjects. They received no training, but were given instructions on the matching procedure to be employed and an opportunity to familiarize themselves thoroughly with the apparatus and procedure before participating in the experiments.

Apparatus. Temperature stimuli were produced using an aluminum temperature column (Figure 2). The column was a solid rectangular $0.9 \times 0.1 \times 0.1 \mathrm{~m}$ block positioned vertically in a wooden frame in front of the observer. The frame also contained two thermostatically controlled water baths, which maintained the two ends of the column at constant temperatures. A cooling element in the water bath attached to the lower end of the column ensured that heat introduced at the upper end could be continuously and efficiently removed from the lower end. With the heat exchangers built into its ends, the column's effective length was reduced to $0.7 \mathrm{~m}$. With appropriately set upper and lower temperatures, a stable and almost linear temperature gradient could be established along the column. For the matching experiments described below, the gradient limits were set by upper and lower temperatures of $47^{\circ}$ and $10^{\circ} \mathrm{C}$, respectively. Details of the physical factors that determined the range of operation of the temperature column are discussed in Tritsch (1986).

On each side of the observer was a large brass adaptation thermode $(100 \times 80 \mathrm{~mm})$ to be used for the fingers of the left and right hands. The thermodes were maintained at steady temperatures $\left( \pm 0.1^{\circ} \mathrm{C}\right)$ through the use of thermostatic or cryothermostatic water baths. The surface temperatures of the thermodes, the end temperatures of the column, the air temperature, and the skin temperature at the tip of the observer's left-hand little finger were continuously monitored, using the digital thermometer described in Experiment 1.

Procedure. The observers sensed temperature stimuli by actively touching the vertical sides of the temperature column. The stimulus area was the volar surface of the medial and distal phalanges of the index and middle fingers of each hand. The observer was ignorant of the actual temperature at any particular place on the column. A movable pointer indicated where he/she was to place his/her fingers for the test stimulus; he/she then matched the temperature sensation produced by the stimulus using the fingers of the other hand. The experimenter did not directly measure the temperature of the matching stimulus found by the observer. Instead, the position was noted and converted into a corresponding temper-

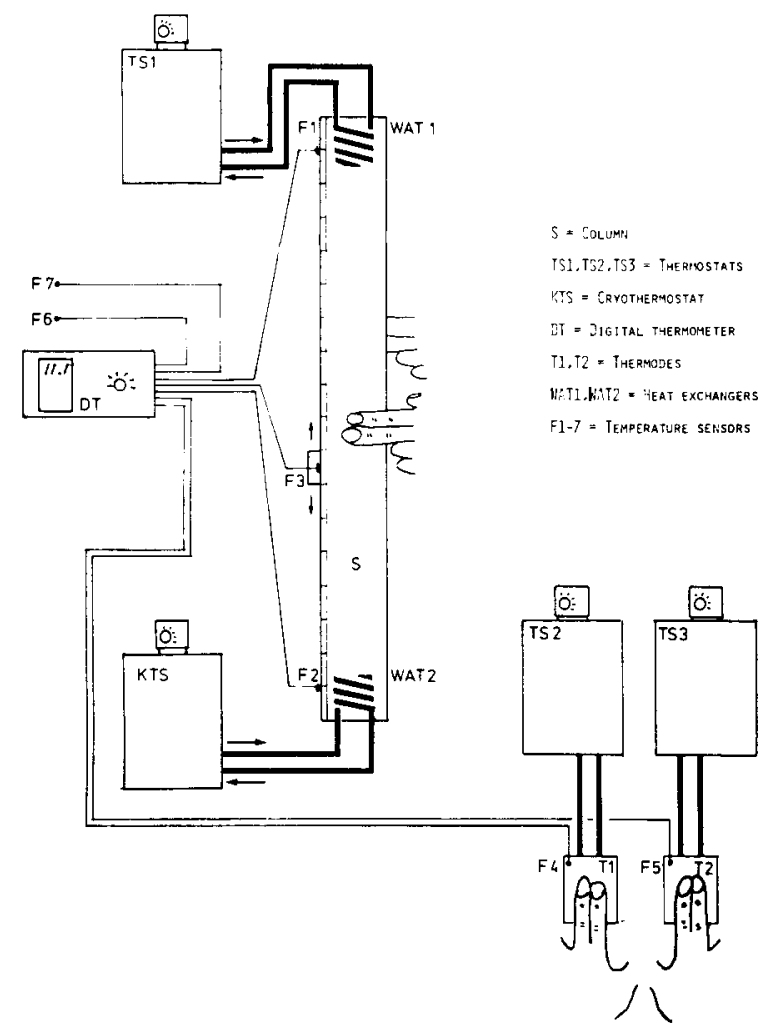

Figure 2. Apparatus used for dichiric matching.

ature by using a calibration curve. Reliability of this method was ensured by the good stability displayed by the temperature gradient. The calibration curve was measured at $50-\mathrm{mm}$ intervals along the column at the beginning of the series of experiments and was checked at the beginning and end of each experiment.

For dichiric matching, the left side of the temperature column was used for test stimuli and the right side for matching stimuli. The left hand of the observer was thus the test hand, and the right hand was the matching hand. The adaptation temperature $T_{\mathrm{Aa}}$ of the thermode reserved for the matching hand was kept at $32^{\circ} \mathrm{C}$ throughout the experiments, whereas that for the test hand could be varied between $15^{\circ}$ and $40^{\circ} \mathrm{C}$. It should be noted that because of the unchanging adaptation temperature of the matching hand, the sensations experienced with it effectively served as a "standard" against which sensations on the test hand could be measured. The choice of $32^{\circ} \mathrm{C}$ as the adaptation temperature for the matching hand was made for several reasons. In addition to being close to average skin temperature under normal conditions, it also produces, in most people, a sensation close to that of "indifference" and permits sensations of both cold and warmth to be generated by a range of object temperatures that is sufficient to ensure accuracy in matching.

For each test, the experimenter set the pointer on the left side of the column at the appropriate test temperature and the observer repeatedly touched the place indicated with the defined stimulus area of the test hand. The observer then immediately began to search with the (differently adapted) matching hand for a place on the right side of the column that produced the same sensation. Care was taken by the experimenter to ensure that the observers all used the same "tapping" technique (an approximately 2-sec contact alternating with a 2-sec removal of the fingers) for both hands. The observers were also instructed to keep the fingers in a straight, closed, and horizontal position. To determine and record the matching posi- 
tion on the column that the observer chose, the experimenter used the border between the two fingers as a reference line. If the observer failed to match the test stimulus within about $15 \mathrm{sec}$, the test was repeated later.

A single experiment involved a fixed setting of $T_{\mathrm{Ap}}$, the adaptation temperature of the test hand. A $15-\mathrm{min}$ preadaptation period for both hands was followed by the test sequence. A set of 9 or (depending on the chosen $T_{\mathrm{Ap}}$ ) 10 different test temperatures, $T_{\mathrm{Op}}$, was presented twice, with the temperatures within the set always in random order. Between each test, both hands were readapted for $2 \mathrm{~min}$. Altogether, six adaptation temperatures, $T_{\mathrm{Ap}}$, were investigated: $15^{\circ}, 20^{\circ}, 25^{\circ}, 30^{\circ}, 35^{\circ}$, and $40^{\circ} \mathrm{C}$. The test temperatures used were $12^{\circ}, 15^{\circ}, 20^{\circ}, 25^{\circ}, 30^{\circ}, 35^{\circ}, 37^{\circ}, 40^{\circ}$, and $42^{\circ} \mathrm{C}$. For a given change in temperature, the change is greater in the sensation of warmth than in the sensation of cold, so test temperatures were chosen to be closer together in the upper part of their range. In addition, a test temperature $2^{\circ} \mathrm{C}$ higher than the adaptation temperature was always included in the range tested.

The experiment in progress was broken off if the air temperature rose to above $24^{\circ} \mathrm{C}$ or the skin temperature measured on the observer's little finger revealed any sudden disturbance in peripheral blood circulation. Replicate experiments that swapped left and right hands were not necessary, since, in principle, there is no difference between variation due to observer differences and that due to differences between an individual observer's hands. Spot checks of individual observers and examination of the matching data itself (see below) showed that there was no systematic bias between left and right hands.

\section{Results and Discussion}

Figure 3 shows the results for $T_{\mathrm{Ap}}=20^{\circ} \mathrm{C}$ in diagrammatic form, and reveals the basic pattern seen at all adaptation temperatures. Veridical perception of object temperature, judged according to the criterion $T_{\mathrm{Oa}}=T_{\mathrm{Op}}$, could be demonstrated at test temperatures that were much higher than the adaptation temperature of the test hand. When test and adaptation temperatures were close

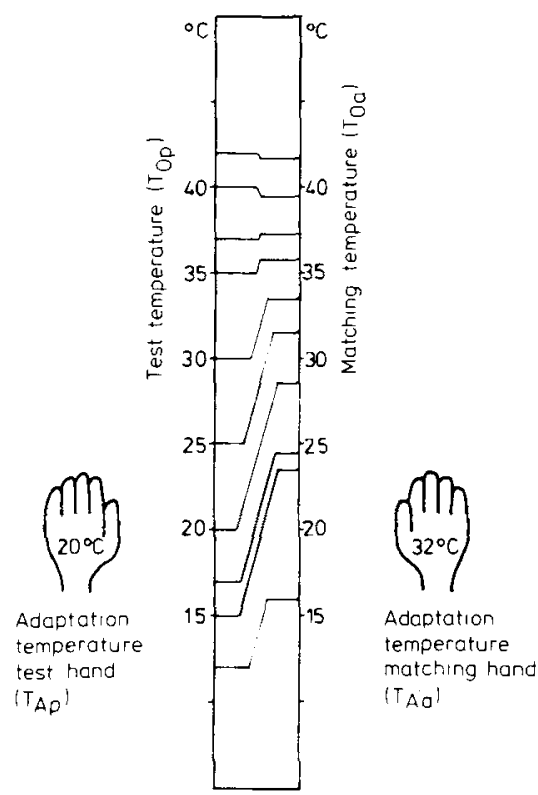

Figure 3. Temperature matches at $T_{\mathrm{Ap}}=20^{\circ} \mathrm{C}$. Slanting lines connect test temperatures to the matching temperatures chosen by the observers. together, the deviation from veridical perception was considerable. When the test temperature was lower than the adaptation temperature, this deviation was reduced. The results shown in this figure, as well as in all the following ones, are arithmetic means of all observers. Clear differences existed among the observers in terms of both the degree to which sensations of cold or warmth faded during preadaptation and in the reliability of their matches. However, the effect of adaptation temperature on objecttemperature perception was the same in all observers.

In Figure 4, matching temperatures are plotted against test temperatures for each of the adaptation temperatures used. Each plot includes two reference lines calculated according to theoretical predictions. The $c=1$ line shows the veridical perception of object temperature, that is, $T_{\mathrm{O}}$ $=T_{\mathrm{Op}}$, irrespective of adaptation temperature. The $c=0$ line shows where the results should lie if objecttemperature sensations were determined solely by the temperature change in the skin, that is, by the difference between adaptation temperature and object temperature. Thus, the extent of deviation from veridical perception can be seen from the position of the results in relation to the two reference lines.

The absolute deviation from veridical perception, $f$, which is the difference between test temperature and the corresponding matching temperature as indicated by the vertical distance between the results curve and the $c=1$ line, depends partly on the difference between the adaptation temperatures of the two hands, as would be expected. However, at a given $T_{\mathrm{Ap}}$, this deviation evidently also varies with test temperature. A comparison of the six curves reveals three features shared by the results from all adaptation temperatures: (1) At high and low test temperatures relative to the adaptation temperature of the test hand, the curves lie on or close to the $c=1$ line; in between, they deviate in the direction of the $c=0$ line. (2) The point of maximum deviation lies approximately half-way between the reference lines and occurs at a test temperature close to or identical with the adaptation temperature of the test hand. (3) On each side of this point, the results appear to show an almost straight-line relationship between $T_{\mathrm{Oa}}$ and $T_{\mathrm{Op}}$.

The point of maximum deviation of each curve from the $c=1$ line in Figure 4 provides the value of $f_{\max }$ with which to examine the effect of varying adaptation temperature on the extent of this deviation. Clearly, there should be no deviation, and therefore $f_{\max }$ should be zero when $T_{\mathrm{Ap}}=T_{\mathrm{Aa}}$; to this extent, all values of $f$ in the results depend on the $T_{\mathrm{Aa}}$ chosen for the matching experiments. (Likewise, convergence of the matching data on the $c=1$ line with a decreasing difference between $T_{\mathrm{Ap}}$ and $T_{\mathrm{Aa}}$ shows that there was no systematic bias between the left and right hands of the observers.) However, the variation of $f_{\max }$ with $T_{\mathrm{Ap}}$ around this null position should be independent of the $T_{\mathrm{Aa}}$ employed. Figure 5 shows that $f_{\text {max }}$ is proportional to $T_{\mathrm{Ap}}$ (and therefore also to the difference between $T_{\mathrm{Ap}}$ and $T_{\mathrm{Aa}}$ ) over the entire range of adaptation temperatures investigated. This means that if $T_{\mathrm{Ap}}$ 

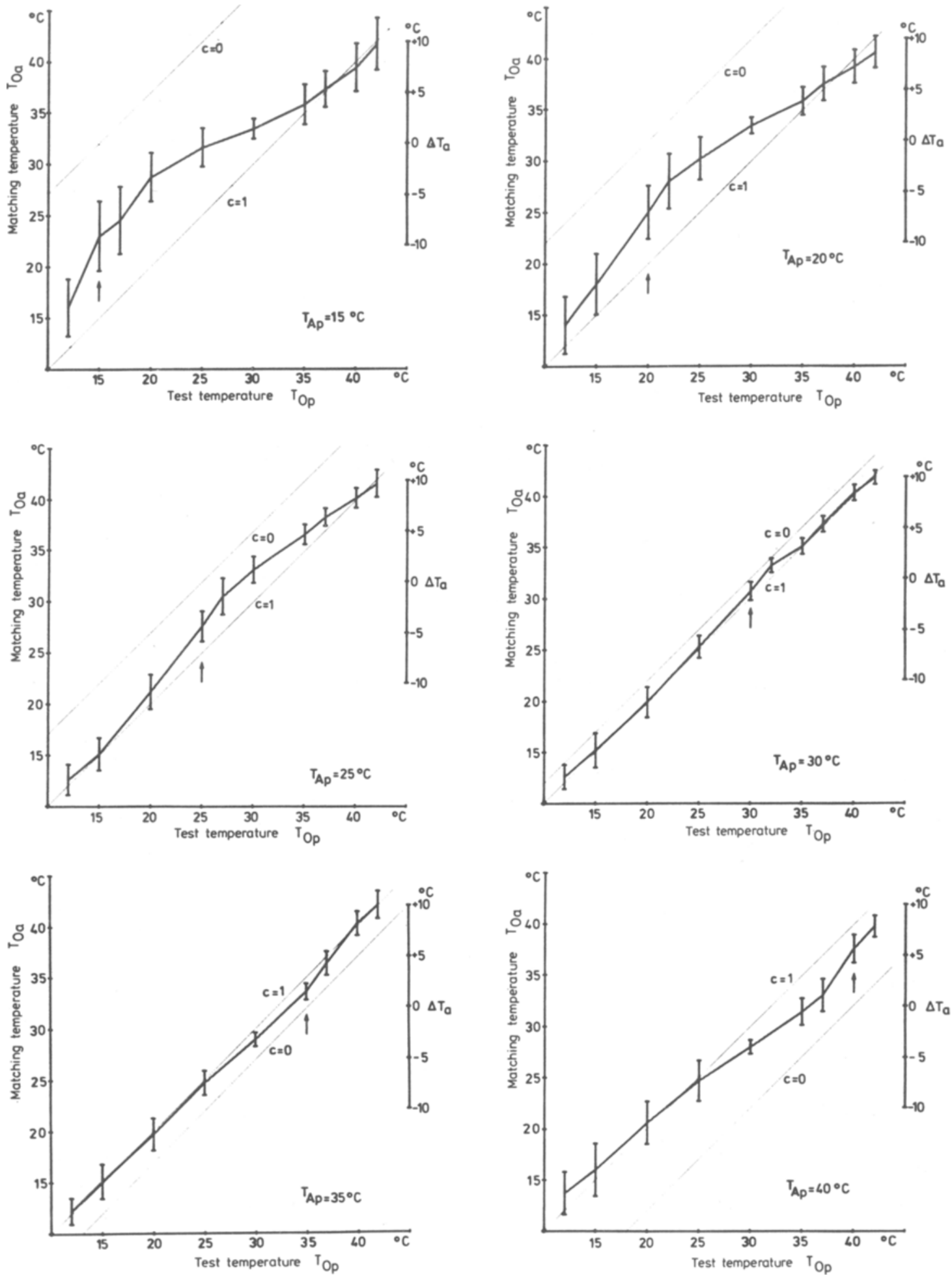

Figure 4. Matching temperature plotted against test temperature for six different adaptation temperatures. Error bars show the standard deviation from the mean. The arrow on each curve indicates the point where test temperature $T_{O p}$ was equal to adaptation temperature $T_{\mathrm{Ap}}$ - 


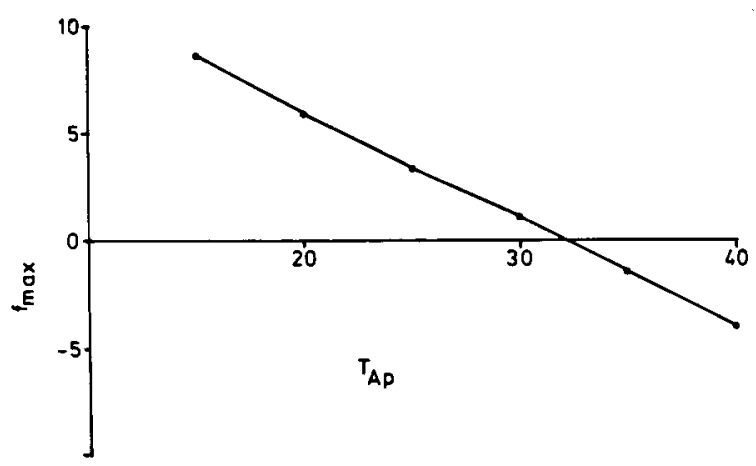

Figure 5. Variation of maximum deviation, $f_{\max }$, with adaptation temperature $T_{\text {Ap }}$.

were the only factor affecting $f$, increasing the difference in adaptation temperature between the two hands would correspondingly increase the sensation difference for a given object temperature. In fact, however, $f$ also varies with the difference between the object temperature tested and the adaptation temperature of the test hand. Since $f_{\max }$ is reached at or near $T_{O p}=T_{A p}$ (arrow in Figure 4), the degree to which veridical perception of object temperature is actually attained must, in fact, be a function of $\Delta T_{p}$, where

$$
\Delta T_{\mathrm{p}}=T_{\mathrm{Op}}-T_{\mathrm{Ap}}
$$

To examine this, the results were corrected for the effects of $T_{\mathrm{Ap}}$ on overall magnitude of the absolute deviation from veridical perception. The result of this correction was the coefficient of deviation, $d$, which is the ratio of the absolute deviation to the difference between testhand and matching-hand adaptation temperatures:

$$
d=\frac{T_{\mathrm{Oa}}-T_{\mathrm{Op}}}{T_{\mathrm{Aa}}-T_{\mathrm{Ap}}} \text {. }
$$

The degree to which veridical perception, $c$, is attained is then defined by

$$
c=1-d .
$$

Figure 7 shows the relationship between $d$ and $\Delta T_{\mathrm{p}}$ for all six adaptation temperatures. The derivation of these plots can be seen in Figure 6. In all cases, $d$ falls from a maximum value of 0.5 near $\Delta T_{p}=0$ toward zero as $\Delta T_{\mathrm{p}}$ increases or decreases from zero. Correspondingly, $c$ rises from 0.5 to 1 . Thus, entirely veridical perception of object temperatures can be assured for objects whose temperature is much lower or higher than that of the skin.

\section{Equal Sensation Curves}

One important reason for selecting $32^{\circ} \mathrm{C}$ as the adaptation temperature for the matching hand was that this temperature provides the nearest approach to an indifferent sensation under completely adapted conditions. Increases and decreases in temperature from this level result, respec- tively, in increasing warmth and increasing cold. Hence $\Delta T_{\mathrm{a}}$, defined by

$$
\Delta T_{\mathrm{a}}=T_{\mathrm{Oa}}-T_{\mathrm{Aa}}
$$

and shown as the right-hand ordinate in Figure 4, provides a measure of the warmth sensation in its positive range and of the cold sensation in its negative range. Since a given value of $\Delta T_{\mathrm{a}}$ corresponds to the same sensation under all conditions of test-hand adaptation, such values can be used to construct equal-sensation curves, even though the scalar relationship between sensation and $\Delta T_{\mathrm{a}}$ is unknown. The various combinations of adaptation temperature and test temperature, which all produce the same sensation in the test hand, can be derived from Figure 4 by taking the points at which the curves intersect given values on the $\Delta T_{\mathrm{a}}$ axis. The resulting equal-sensation curves (Figure 8) show that strong sensations produced by object temperatures (e.g., when $\Delta T_{\mathrm{a}}$ is +7.5 or -15 ) are relatively resistant to the effects of adaptation temperature and therefore allow veridical perception of object temperature, while sensations that are weak (e.g., $\Delta T_{\mathrm{a}}$ $=-3$ ) provide no reliable guide to the temperature of the object.

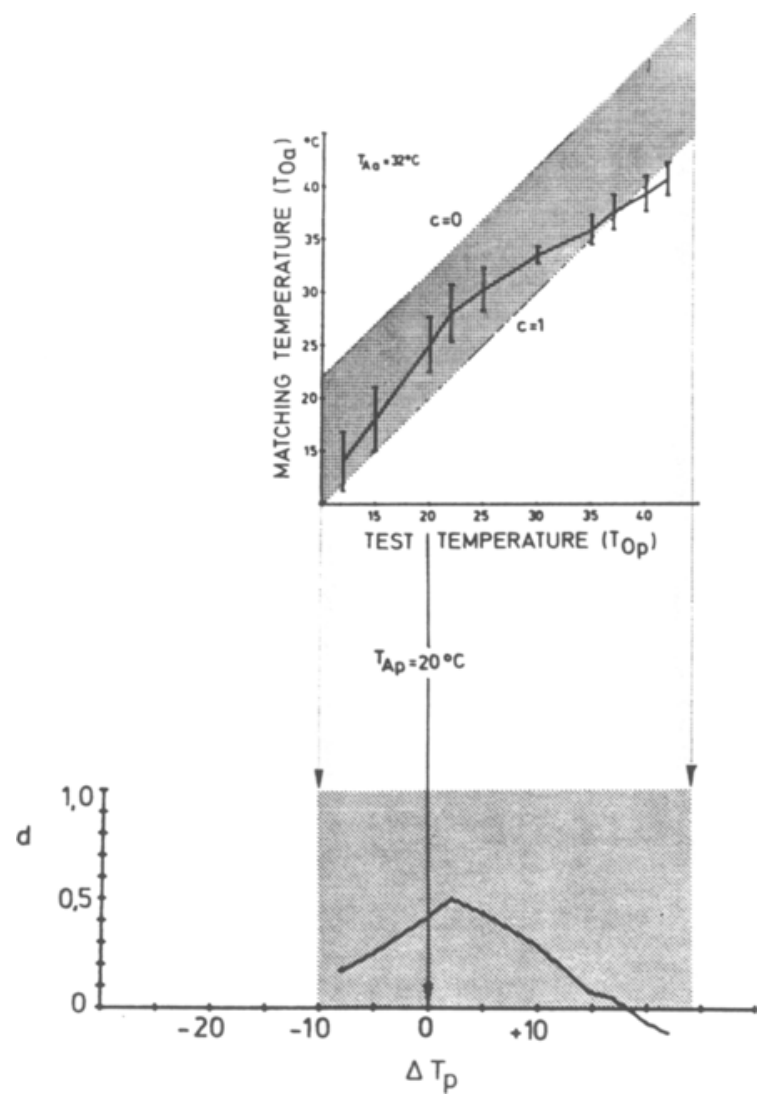

Figure 6. Derivation of curves showing $d$ and $\Delta T_{p}$ from the results shown in Figure 4. The abscissa of the lower plot is adjusted horizontally so that $\Delta T_{p}=0$ lies directly below the value of $T_{O p}$ in the upper plot equal to $T_{\mathrm{Ap}} \cdot d=1$ corresponds to the vertical distance between the $c=0$ and $c=1$ lines. 


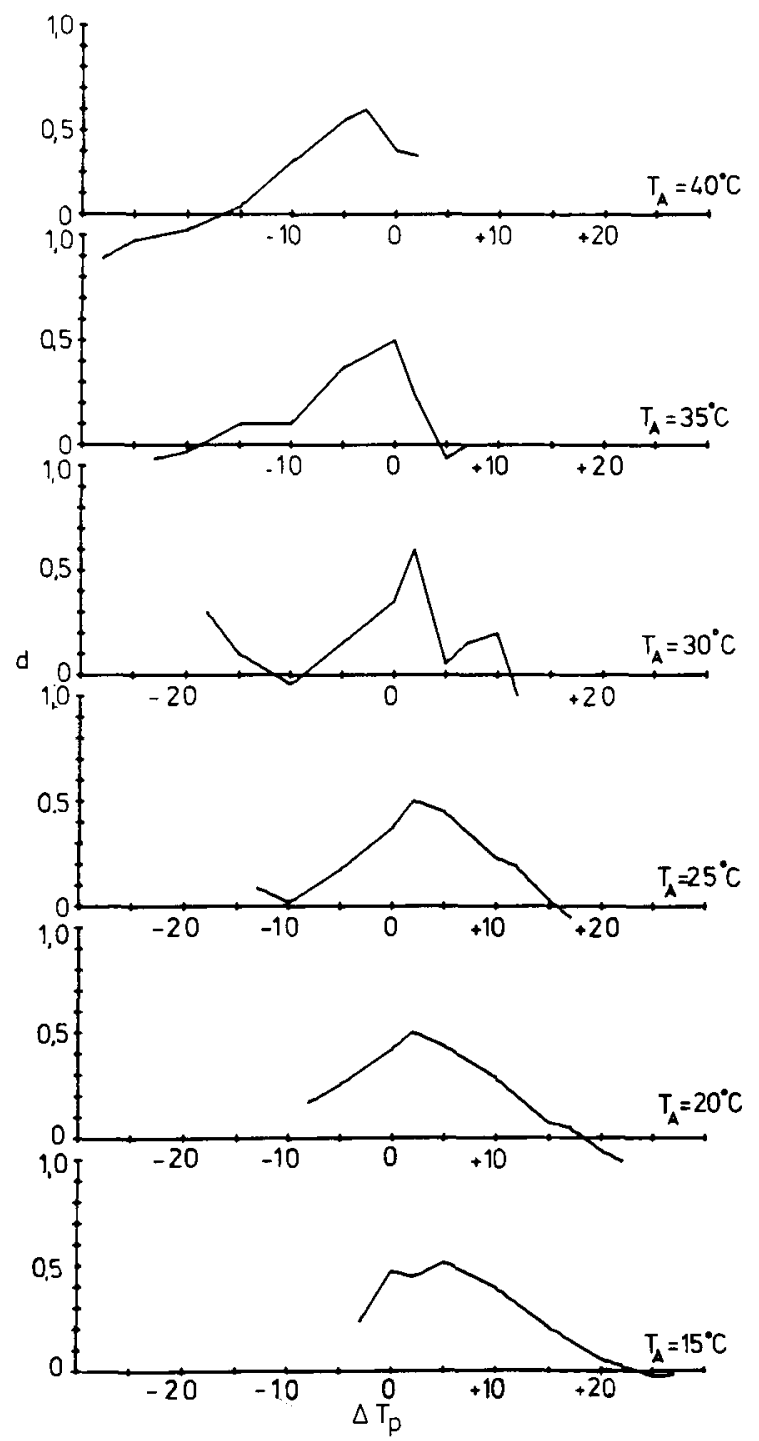

Figure 7. Coefficient of deviation $d$ plotted against difference $\Delta T_{p}$ between test temperature and adaptation temperature. The six adaptation temperatures are shown above one another. Note the close resemblance among all the curves.

The spacing of the equal-sensation curves suggests that warmth sensitivity should increase and cold sensitivity should decrease with increasing adaptation temperature. Using adaptation temperatures down to $28^{\circ} \mathrm{C}$, Kenshalo (1976) measured the increment threshold for warmth and cold in human observers, and found a similar dependency upon adaptation temperature. The equal-sensation curves continue to show closer spacing even at an adaptation temperature of $15^{\circ} \mathrm{C}$.

\section{CONCLUSIONS}

The present experiments demonstrate that for every adaptation temperature examined, object-temperature perception approaches the veridical as the temperature dif- ference between skin and object increases. Object temperatures could always be found for which perception was entirely veridical. Furthermore, since $d$, the coefficient of deviation from veridical perception, was found never to exceed 0.5 , it is clear that object-temperature sensations cannot provide a consistent report about temperature change in the skin. Although such sensations include contributions of both object temperature and change in skin temperature, the relative proportions of which depend on $\Delta T_{p}$, it is primarily the physical temperature of the object with which they are most strongly correlated.

Do these features of object-temperature perception reflect simply the properties of the adequate stimulus for cutaneous, specific thermoreceptors? Both cold and warmth receptors display a dynamic response to a temperature change in the skin, which then adapts to a static response to a maintained temperature (see Hensel, 1981, for review). Thus, the receptors do convey information about both constant and changing temperatures, as would be expected if they are the basis for the features of temperature sensation reported here.

The attempt to explain object-temperature perception in terms of the receptor response presents a difficulty, however. Obviously, the receptors cannot measure the temperature of an object directly; they can only measure the temperature where they are situated, as much as $0.6 \mathrm{~mm}$ below the surface of the skin. Because of the skin's thermally insulating properties, when an object that is colder or warmer than the skin is touched, the temperature in the skin at the depth of the receptors requires more than $10 \mathrm{sec}$ to reach its new level, as shown by the measurements Hensel (1952) obtained by using intracutane-

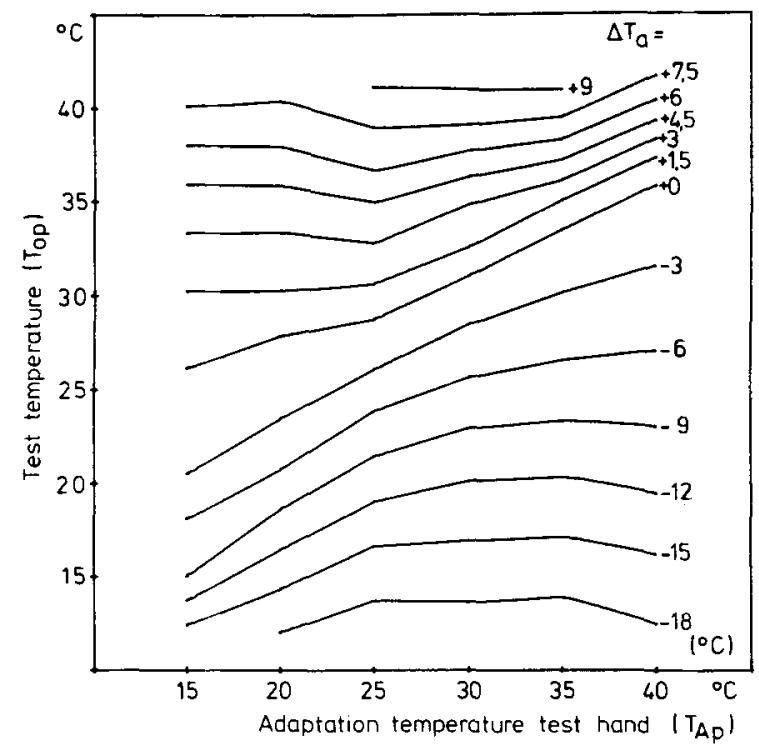

Figure 8. Equal-sensation curves drawn for chosen values of $\Delta T_{\mathrm{a}}$ (shown to the right of each curve). The curves show how test temperature and adaptation temperature trade off to produce a given sensation. 
ously inserted thermoelements. The cold or the warmth sensation, however, rises to a steady level within as little as 1 or $2 \mathrm{sec}$. This means that, within the short time period needed for sensation, the temperature receptors, instead of measuring the final temperature reached, must respond, primarily dynamically, to the rate and extent of temperature change. Now the magnitude of this change is determined as much by the adaptation temperature of the skin as by the object temperature. The physical stimulus for the receptors is therefore not an object temperature at all, but, rather, a temperature change that in itself is not even uniquely related to the object temperature. This points to the presence of some mechanism that is able to extract information about object temperature by using both the previously adapted static receptor response and the dynamic response to temperature change.

Figure 9 diagrams the simplest possible mechanism. The assumption is that adaptation temperature, $T_{\mathrm{A}}$, and change in skin temperature at the receptors, $\Delta T^{* *}$, signaled by the static and dynamic discharge of the receptors, respectively, and that they are processed in parallel and then summed to give the object temperature $T_{0}$ as output. Since warmth and cold are not only distinct qualities of sensation, but also are associated with two different receptor populations, one must suppose that each has an appropriate offset for $T_{\mathrm{A}}$ and sign for $\Delta T^{* *}$. This mechanism is based on the fact that object temperature can be derived by summing $T_{\mathrm{A}}$ and $k \cdot \Delta T^{* *} / I(t)$, the latter term being the time-sampled skin-temperature change weighted by a constant, $k$, representing the heat-transfer properties of the object (see Appendix). Since the proposed mechanism has no input for $k$, its output will display a bias dependent on the object material used.

In terms of the matching experiments, for a given material this mechanism would produce an absolute deviation $f$ proportional to $T_{\mathrm{Aa}}-T_{\mathrm{Ap}}$. This corresponds to the results shown in Figure 5. A second prediction, that $f$ will vary with the heat-transfer properties of the material used, is easily verifiable but has not yet been quantitatively in-

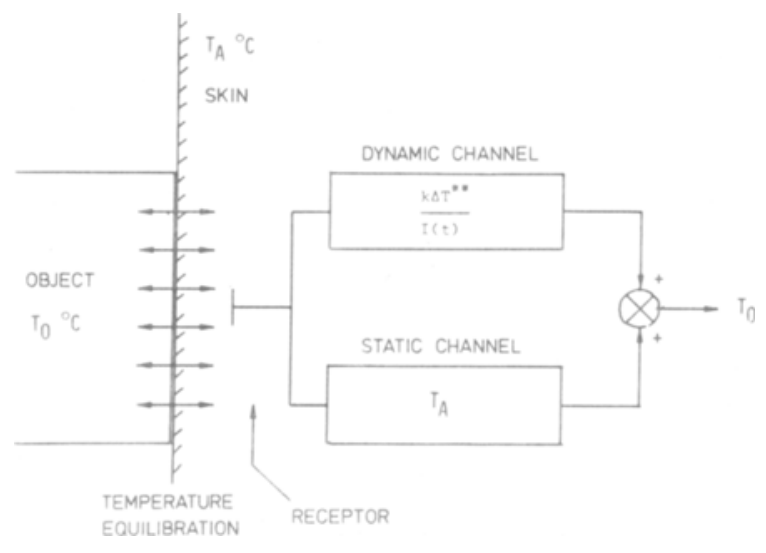

Figure 9. Mechanism for veridical perception of object temperature based on summation of outputs from static and dynamic chanriels. $k$ corresponds to the heat-transfer properties of the object. vestigated. The third prediction is that the coefficient of deviation $d$ will not vary with $\Delta T_{\mathrm{p}}$. As Figure 7 shows, this prediction fails. An interaction between static and dynamic channels prior to their summation could be included in the mechanism in order to satisfy the requirement that $d$ increase as $\Delta T_{\mathrm{p}}$ approaches zero. A multiplicative interaction would allow the weighting of the two channels to be varied according to the current combination of adaptation temperature and temperature change. The degree to which veridical perception is approached would depend on $\Delta T_{\mathrm{p}}$ if, for example, the gain in the dynamic channel were controlled by $T_{\mathrm{A}}$.

What conclusions about the biological function of object-temperature perception can be drawn from these experiments? One important function specifically of object-temperature sensations may be simply to sense the physical temperature of objects in the surroundings. Since this temperature is not directly sensed by the receptors (see above), it would be reasonable to describe veridical perception of object temperature as a case of perceptual constancy, analogous to that found in other sensory modalities (Campenhausen, 1981; Graham \& Brown, 1965; Hochberg, 1971; Julesz, 1969). Indeed, the derivation of $c$, the measure of the degree to which veridical perception is attained, makes it formally equivalent to the Brunswik ratio used in studies of constancy (Brunswik, 1934; Teuber, 1960). It is arguable, however, that the concept of perceptual constancy is inappropriate to the temperature domain. Sensations of warmth and cold have been shown under several experimental conditions to exhibit considerable spatial summation (J. C. Stevens \& Marks, 1971, 1979). Regional variation in sensitivity (J. C. Stevens, 1979; J. C. Stevens, Marks, \& Simonsen, 1974) also does not appear to be consistent with a constancy approach to temperature sensation. Furthermore, a theory of perceptual constancy has difficulties in taking account of the influence of object material on object-temperature sensation (see Appendix).

The absence of consistent information about temperature change provided by object-temperature sensations leads to one definite, although negative, conclusion. Temperature change in the skin is not, itself, a matter of primary interest for the human subject when his skin comes into physical contact with an object. Since this contradicts a commonly held assumption, it is worth considering the reasons. A list of possible functions of object-temperature sensations should include identification of object temperature per se, prevention of skin damage, measurement of the thermal load on the organism, and recognition of object material. Of these, only the last requires that temperature change determine sensation (see Appendix). Note that temperature change in the skin provides no information about the thermal load on the organism. This depends, rather, on the rate of heat transfer across the skin following temperature equilibration at the skin surface, that is, the difference between core temperature and object temperature. At the most, temperature change in the skin informs the organism of a change in thermal load. 
Of course, temperature sensations occur in many stimulus contexts differing sharply from those considered in this study, many of them of particular importance for thermoregulation. In these cases, temperature change in the skin may sometimes be of greater importance. Indeed, the neural mechanisms of temperature sensation possibly reflect the operation of various opposing biological requirements.

Such opposing needs may, indeed, provide a plausible explanation for the deviation from veridical objecttemperature perception found at low (absolute) values of $\Delta T_{\mathrm{p}}$. Optimal conditions for distingishing different object materials from one another would obviously be present if temperature sensations were determined by temperature change alone (see Appendix). Since, under these conditions, veridical object-temperature perception would be impossible, evolution may have favored a balancing out of what are necessarily conflicting demands on the sensory system. The sacrifice of veridical objecttemperature perception at low $\Delta T_{\mathrm{p}}$ leads to a shift from the $c=1$ line toward the $c=0$ line (Figure 4), so that there is, in fact, an increased weighting of temperature change relative to adaptation temperature in producing temperature sensations. This sacrifice may be restricted to object temperatures close to skin temperature, because there is greater survival value attached to signaling the presence of very warm or very cold objects in terms of their physical temperature, especially where skin damage may occur.

Finally, it should be noted that the intensity of sensation itself provides information concerning the degree to which object temperature is veridically perceived. The equal-sensation curves show that an observer may assume that when he touches an object that produces intense cold or warmth he is sensing its actual temperature. Weaker sensations may be interpreted as being biased by the material composition of the object.

\section{REFERENCES}

Brunswik, E. (1934). Wahrnehmung und Gegenstandswelt. Leipzig: Deuticke.

Campenhausen, C. von. (1981). Die Sinne des Menschen. Stuttgart: Thieme.

FREY, M. voN. (1904). Vorlesungen über Physiologie. Berlin: Springer.

Goldscheider, A. (1926). Thermoreceptoren. In A. Bethe (Ed.), Handbuch der normalen und pathologischen Physiologie (Vol. 11, pp. 131164). Berlin: Springer.

Graham, C. H., Brown, J. L. (1965). Color contrast and color appearances: Brightness constancy and color constancy. In C. H. Graham (Ed.), Vision and visual perception (pp. 452-478). New York: Wiley.

Gröber, H., Erk, S., \& Grigull, U. (1955). Die Grundgesetze der Warrmeübertragung. Berlin: Springer.

HahN, H. (1929). Ein neues sinnesphysiologisches Gesetz. Klinische Wochenschrift, 8, 1016.

HARDY, J. D., \& OPPEL, T. W. (1937). Studies in temperature sensation: III. The sensitivity of the body to heat and the spatial summation of the end organ responses. Journal of Clinical Investigations, 16, 533-540.

Hensel, H. (1952). Physiologie der Thermoreception. Ergebnisse der Physiologie, 47, 166-368.
Hensel, H. (1981). Thermoreception and temperature regulation. London: Academic Press.

Hering, E. (1877). Grundzüge einer Theorie des Temperatursinns. Sitzungsberichte der Akademie der Wissenschaften in Wien, Abteilung $3,75,101-135$.

Hö̈ER, R. (1919). Lehrbuch der Physiologie des Menschen. Berlin: Springer.

HochberG, J. (1971). Perception: I. Color and shape. In J. W. Kling \& L. A. Riggs (Eds.), Woodworth \& Schlosberg's experimental psychology (pp. 395-474). London: Methuen.

JuLESZ, B. (1969). Optical-constancy phenomena. In W. Reichardt (Ed.), Processing of optical data by organisms and by machines (pp. 417430). New York: Academic Press.

Kenshalo, D. R. (1971). The cutaneous senses. In. J. W. Kling \& L. A. Riggs (Eds.), Woodworth \& Schlosberg's experimental psychology (pp. 117-168). London: Methuen.

KenSHALO, D. R. (1976). Correlations of temperature sensitivity in man and monkey, a first approximation. In Y. Zotterman (Ed.), Sensory functions of the skin in primates. Oxford: Pergamon Press.

MARKs, L. E., \& STEvENS, J. C. (1973). Spatial summation of warmth: Influence of duration and configuration of the stimulus. American Journal of Psychology, 86, 251-267.

Rózsa, A. J., \& Kenshalo, D. R. (1977). Bilateral spatial summation of cooling of symmetrical sites. Perception \& Psychophysics, 21, $455-462$.

STEVENS, J. C. (1979). Variations of cold sensitivity over the body surface. Sensory Processes, 3, 317-326.

Stevens, J. C., Marks, L. E. (1971). Spatial summation and the dynamics of warmth sensation. Perception \& Psychophysics, 9 , 391-398.

Stevens, J. C., \& MARKs, L. E. (1979). Spatial summation of cold. Physiology \& Behavior, 22, 541-547.

Stevens, J. C., Marks, L. E., \& Simonsen, D. C. (1974). Regional sensitivity and spatial summation in the warmth sense. Physiology \& Behavior, 13, 825-836.

Stevens, S. S. (1971). Issues in psychophysical measurement. Psychological Review, 78, 426-450.

Teuber, H.-L. (1960). Perception. In J. Field, H. W. Magoun, \& V. E. Hall (Eds.), Handbook of Physiology: Vol. III. Neurophysiology (pp. 1595-1668). Washington: American Physiological Society.

TRITSCH, M. F. (1986). Zur Psychophysik der Temperaturwahmehmung: Die Erfassung von Information uber Temperatur und Stoff. Doctoral dissertation, Johannes Gutenberg University, Mainz.

WEBER, E. H. (1846). Der Tastsinn und das Gemeingefühl. In R. Wagner (Ed.), Handwörterbuch der Physiologie (Vol. III.2, pp. 481-588). Braunschweig: Vieweg.

\section{NOTES}

1. Although first published by Hering (1877), this experiment is often erroneously attributed to the 19th century German physiologist E. H. Weber. So far as the author can tell, the earliest occurrence of this mistaken attribution in the German literature occurs in Höber (1919).

2 . When both hands were immersed simultaneously, minor assimilation and contrast effects were noted. These effects were not present when a thermode was used in place of the test bath.

3. Range effects (S. S. Stevens, 1971) in the second series of Experiment 1 were excluded by statistical comparison of the magnitude estimates for the first and third groups of stimulus presentations in that series. No statistically significant differences could be detected.

\section{APPENDIX}

Figure A1 shows, in simplified terms, the process of temperature equilibration that occurs when the plane surface of a block of glass is brought into contact with the surface of the skin (Gröber, Erk, \& Grigull, 1955). At the assumed depth of the 


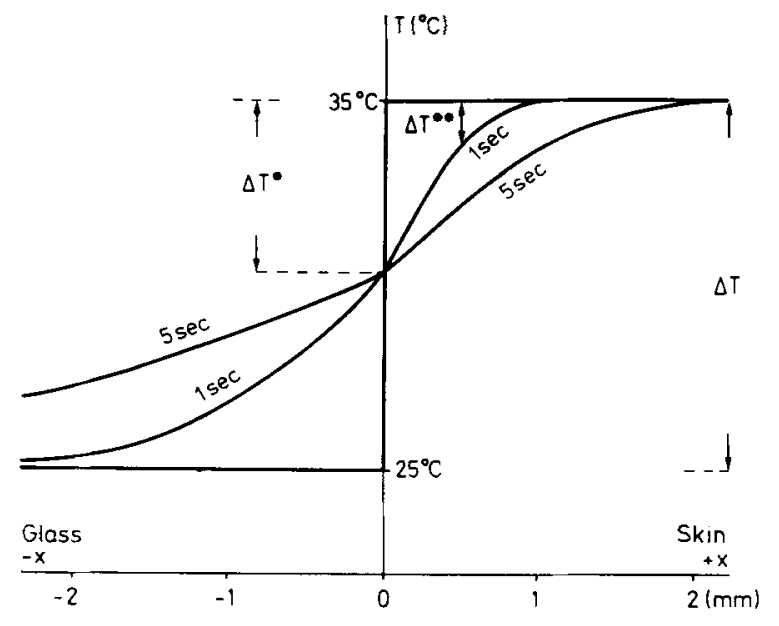

Figure A1. Temperature equilibration in space and time during contact of the skin $\left(35^{\circ} \mathrm{C}\right)$ with a block of glass $\left(25^{\circ} \mathrm{C}\right)$, calculated for a set of simplifying conditions. Temperature is plotted on the vertical axis, depth in skin (right) and glass (left) on the horizontal axis. The three emphasized lines show the temperature distribution at time 0 and after 1 and 5 sec.

receptors (in this case $0.5 \mathrm{~mm}$ ), the temperature change $\Delta T^{* *}$, which occurs during the time period $t$ after the two surfaces are brought together, is given by

$$
\Delta T^{* *}=k^{-1} \cdot \Delta T \cdot I(t),
$$

where $\Delta T$ is the difference between the object temperature $T_{0}$ and the adaptation temperature of the skin $T_{\mathrm{A}}, I(t)$ has the form of a cumulative Gaussian, and $k$ depends on the heat transfer properties of the material concerned, in this case glass. It obviously follows that

$$
T_{\mathrm{O}}=T_{\mathrm{A}}+\frac{k \cdot \Delta T^{* *}}{l(t)} .
$$

This relationship allows the absolute temperature of an object to be derived, for a given material, from the two inputs $\Delta T * *$ and the adaptation temperature by any mechanism able to carry out the appropriate transformations, using $k$ and $I(t)$.

Due to their differing heat-transfer properties, materials vary in the degree to which they cause an alteration in skin temperature when touched. It can be shown that when two materials differing in temperature are brought into contact, as in Figure $\mathrm{Al}$, the temperature that appears almost immediately at the junction of the two objects persists during the entire process of temperature equilibration (Gröber et al., 1955). This temperature, which results in a temperature change of $\Delta T^{*}$ at the skin surface, is dependent on the heat-transfer properties of the skin (which remain relatively constant) and the object. $\Delta T^{* *}$ for any given time after contact is, in turn, directly proportional to $\Delta T^{*}$, allowing it to provide information concerning the material.

\section{GLOSSARY}

$\begin{array}{ll}T_{\mathrm{O}} & \text { Object temperature } \\ T_{\mathrm{A}} & \text { Adaptation temperature } \\ \Delta T & T_{\mathrm{O}}-T_{\mathrm{A}} \\ \Delta T^{*} & \text { Temperature change at skin surface } \\ \Delta T^{* *} & \text { Temperature change at level of receptors }\end{array}$

\section{Experiment 1}

$T_{\mathrm{Al}} \quad$ Adaptation temperature, left hand

$T_{\mathrm{Ar}} \quad$ Adaptation temperature, right hand

$T_{\mathrm{O}_{\mathrm{r}}} \quad$ Object-temperature stimulus, right hand

$E \quad$ Subjective estimate of magnitude of warmth

\section{Experiment 2*}

$T_{\text {Aa }} \quad$ Adaptation temperature, matching hand

$T_{\text {Ap }} \quad$ Adaptation temperature, test hand

$T_{\text {Oa }} \quad$ Matching temperature

$T_{O p} \quad$ Test temperature

$\Delta T_{\mathrm{a}} \quad T_{\mathrm{Oa}}-T_{\mathrm{Aa}}$

$\Delta T_{\mathbf{p}} \quad T_{\mathbf{O p}}-T_{\text {Ap }}$

$f \quad$ Absolute deviation from veridical perception

$d \quad$ Coefficient of deviation from veridical perception

$c$ Degree of veridical perception $(1-d)$

*The subscripts a and $p$ refer to the German words abgleichen (match) and prüfen (test).

(Manuscript received December 19, 1986; revision accepted for publication October $29,1987$. ) 